\title{
Vibration Prediction in Switched Reluctance Motors With Transfer Function Identification From Shaker and Force Hammer Tests
}

\author{
Zhangjun Tang, Member, IEEE, Pragasen Pillay, Senior Member, IEEE, and \\ Avoki M. Omekanda, Senior Member, IEEE
}

\begin{abstract}
A vibration prediction model for the switched reluctance motor is constructed in this paper. Shaker and force hammer tests for vibration measurement are used for measuring crucial parameters like modal frequency and damping ratio for the transfer function. A detailed lookup table of normal force versus phase current and rotor angle is constructed based on finite-element calculations. The model is then verified by experiments, with acceptable accuracy.
\end{abstract}

Index Terms-Finite-element (FE) calculation, hammer excitation, normal force, shaker, sinusoidal excitation, switched reluctance motor (SRM), vibration.

\section{INTRODUCTION}

$\mathbf{I}$ T IS ALREADY accepted that the vibrations in switched reluctance motors (SRMs) are caused primarily by the ovalizing deformation of the stator lamination stack due to its radial magnetic attraction to the rotor [1], [2]. Currently, there is work being done on the determination of resonant frequencies [3], [4], mode shapes [5]-[7], and testing [8]-[10]. However, the prediction of vibration deserves some attention.

This paper will make it possible to predict the vibration in the SRM, using the vibration transfer function obtained from shaker and impulse hammer tests, together with a normal force lookup table from finite-element (FE) calculations.

Fig. 1 shows the flowchart for vibration prediction modeling and verification. The final goal is to compare the predicted and measured accelerations. As can be seen from the flowchart, there are two paths: one for predicted acceleration and the other one for measured acceleration.

The most important and difficult path is the "Predicted Acceleration," where a lot of work has to be done; this requires shaker and force hammer tests, parameter determination, transfer function modeling, FE method (FEM) normal force calculation, lookup table construction, running SRM current

Paper IPCSD 03-044, presented at the 2002 Industry Applications Society Annual Meeting, Pittsburgh, PA, October 13-18, and approved for publication in the IEEE TRANSACTIONS ON INDUSTRY APPLICATIONS by the Electric Machines Committee of the IEEE Industry Applications Society. Manuscript submitted for review July 1, 2002 and released for publication April 30, 2003. This work was supported by Delphi Research Labs, Shelby Township, MI.

Z. Tang is with Stryker Instruments, Kalamazoo, MI 49011 USA (e-mail: ztang@inst.strykercorp.com).

P. Pillay is with the Department of Electrical and Computer Engineering, Clarkson University, Potsdam, NY 13699 USA (e-mail: pillayp@ clarkson.edu).

A. M. Omekanda is with Delphi Research Labs, Shelby Township, MI 48315

USA (e-mail: avoki.omekanda@ delphi.com).

Digital Object Identifier 10.1109/TIA.2003.814558

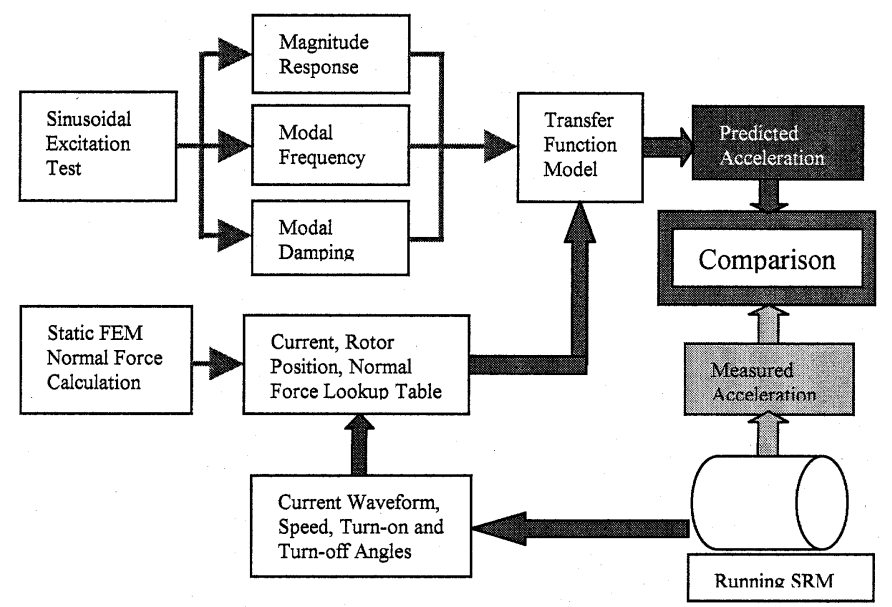

Fig. 1. Flowchart of vibration prediction modeling and verification.

and acceleration measurements, etc. Firstly, shaker tests are done for SRM vibrations. Three parameters are needed for the transfer function model of each resonant frequency, and they are then used to construct the transfer function model in SIMULINK. Secondly, intensive FEM calculations are done using ANSYS to construct a lookup table of SRM phase current, rotor position, and normal force. Next, the phase current waveform, motor speed, turn-on, and turn-off angles of the running SRM are recorded and then used in the lookup table constructed in the previous step. If the motor is not available, simulated currents from $\psi-i$ data can be used. The actual normal force applied to the stator-rotor pole is calculated while the SRM is running at different speeds. Finally, the calculated normal force data is used in the transfer function to predict the acceleration and, hence, noise.

For the measured acceleration path, several accelerometers are mounted directly on the outer case of the stator. The motor is running and acceleration spectra are recorded under different motor speeds. This is compared with the predicted acceleration with acceptable results.

The paper makes the following assumptions.

1) The mechanical modal transfer function can be represented as the linear superposition of several single-degree-of-freedom (SDOF) systems.

2) The transient effects of the current on normal forces can be ignored.

3) The normal forces concentrate on the stator-rotor pole areas. 


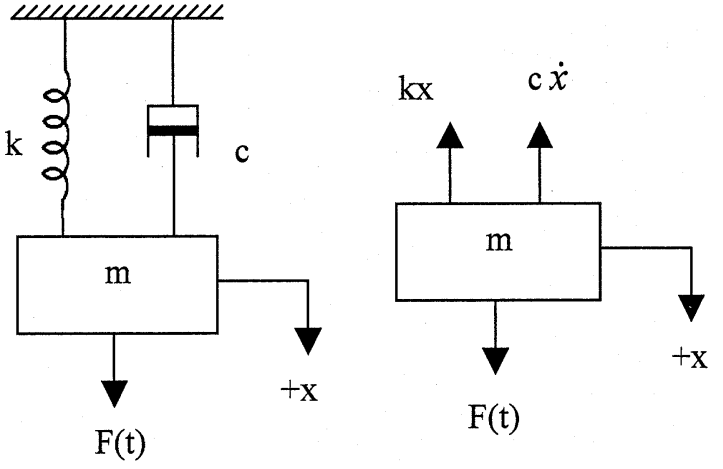

Fig. 2. A spring-mass-damper system.

\section{DeVElopment of ThE TRANSFER FunCtion FOR EACH MODE}

\section{A. Frequency Response Function (FRF) of SRM Vibrations}

From a vibration point of view, the SRM is a complicated multidegree-of-freedom (MDOF) system [11], [12]. However it can be represented as the linear superposition of a finite number of SDOF systems.

Fig. 2 is the model for a force acting on a viscous damped spring-mass system. The equation of motion can be obtained using Newton's second law

$$
m \ddot{x}+c \dot{x}+k x=F(t) .
$$

For $\omega_{n}=\sqrt{k / m}=$ undamped natural frequency, $\zeta=$ $c / 2 m \omega_{n}=c / 2 \sqrt{m k}=$ Damping ratio, and $c / m=2 \zeta \omega_{n}$, the displacement response (Compliance) of the system is

$$
X(s)=\frac{F(s)}{m} \frac{1}{s^{2}+2 \zeta \omega_{n} s+\omega_{n}^{2}} .
$$

Velocity response (Mobility) is

$$
V(s)=s X(s)=\frac{F(s)}{m} \frac{s}{s^{2}+2 \zeta \omega_{n} s+\omega_{n}^{2}} .
$$

Acceleration response (Accelerance) is

$$
a(s)=s^{2} X(s)=\frac{F(s)}{m} \frac{s^{2}}{s^{2}+2 \zeta \omega_{n} s+\omega_{n}^{2}} .
$$

Therefore, the transfer function from force to acceleration is

$$
H(s)=\frac{a(s)}{F(s)}=\frac{1}{m} \frac{s^{2}}{s^{2}+2 \zeta \omega_{n} s+\omega_{n}^{2}} .
$$

For the stator of the SRM, the transfer function can be written as

$$
H(s)=\frac{a(s)}{F(s)} \cong \sum_{i} A_{i} \frac{s^{2}}{s^{2}+2 \zeta_{i} \omega_{n i} s+\omega_{n i}^{2}} .
$$

where $\omega_{n i}$ is the resonant natural frequency of mode $i, \zeta_{i}$ is the damping ratio of mode $i, A_{i}$ is the gain relative to mode $i$, and $\mathrm{s}$ is the Laplace's variable.

The transfer function of the stator vibrations and the forces acting on the stator is composed of a sum of the second-order high-pass filters.

\section{B. Damping Ratio Measurement}

The motion of free vibration of the SODF system with nonzero initial condition can be expressed as $m \ddot{x}+c \dot{x}+k x=0$.
To solve this equation, it can be assumed that the solution is in the form of $x(t)=C e^{r t}$, where $\mathrm{C}$ and $\mathrm{r}$ are undetermined constants. The solution becomes

$$
\begin{aligned}
& x(t)=e^{-\zeta \omega_{n} t} \\
& \quad\left\{x_{0} \cos \sqrt{1-\zeta^{2}} \omega_{n} t+\frac{\dot{x}_{0}+\zeta \omega_{n} x_{0}}{\sqrt{1-\zeta^{2} \omega_{n}}} \sin \sqrt{1-\zeta^{2}} \omega_{n} t\right\} .
\end{aligned}
$$

The constants $(X, \phi)$ and $\left(X_{0}, \phi_{0}\right)$ can be expressed as $X=X_{0}=\sqrt{\left(C_{1}^{\prime}\right)^{2}+\left(C_{2}^{\prime}\right)^{2}}, \phi=\tan ^{-1}\left(C_{1}^{\prime} / C_{2}^{\prime}\right)$ and $\phi_{0}=\tan ^{-1}\left(-C_{2}^{\prime} / C_{1}^{\prime}\right)$, where $C_{1}^{\prime}=x_{0}$ and $C_{2}^{\prime}=\dot{x}_{0}+\zeta \omega_{n} x_{0} / \sqrt{1-\zeta^{2} \omega_{n}}$ for the initial conditions $x(t=0)=x_{0}$ and $\dot{x}(t=0)=\dot{x}_{0}$. The motion described by the solution is damped harmonic motion of angular frequency $\sqrt{1-\zeta^{2}} \omega_{n}$, but because of the factor $e^{-\zeta \omega_{n} t}$, the amplitude decreases exponentially with time. The quantity $\omega_{d}=\sqrt{1-\zeta^{2}} \omega_{n}$ is called the frequency of damped vibration. It can be seen that the frequency of damped vibration $\omega_{d}$ is always less than the undamped natural frequency $\omega_{n}$.

The logarithmic decrement represents the rate at which the amplitude of a free damped vibration decreases. It is defined as the natural logarithm of the ratio of any two successive amplitudes. Let $t_{1}$ and $t_{2}$ denote the times corresponding to two consecutive amplitudes (displacements), measured one cycle apart for an underdamped system. The ratio of the displacements $x_{1} / x_{2}=X_{0} e^{-\zeta \omega_{n} t_{1} \cos \left(\omega_{d} t_{1}-\phi_{0}\right)} / X_{0} e^{-\zeta \omega_{n} t_{2} \cos \left(\omega_{d} t_{2}-\phi_{0}\right)}$ can be formed. However, $t_{2}=t_{1}+\tau_{d}$ where $\tau_{d}=2 \pi / \omega_{d}$ is the period of damped vibration. Hence, $\cos \left(\omega_{d} t_{2}-\phi_{0}\right)=$ $\cos \left(2 \pi+\omega_{d} t_{1}-\phi_{0}\right)=\cos \left(\omega_{d} t_{1}-\phi_{0}\right)$, and the ratio can be rewritten as $x_{1} / x_{2}=e^{-\zeta \omega_{n} t_{1}} / e^{-\zeta \omega_{n}\left(t_{1}+\tau_{d}\right)}=e^{\zeta \omega_{n} \tau_{d}}$. The logarithmic decrement $\delta$ can be obtained as

$$
\delta=\ln \frac{x_{1}}{x_{2}}=\zeta \omega_{n} \tau_{d}=\zeta \omega_{n} \frac{2 \pi}{\sqrt{1-\zeta^{2}} \omega_{n}}=\frac{2 \pi \zeta}{\sqrt{1-\zeta^{2}}}=\frac{2 \pi}{\omega_{d}} \cdot \frac{c}{2 m} .
$$

For small damping, it can be approximated as $\delta \cong 2 \pi \zeta$ if $\zeta \ll 1$.

The logarithmic decrement is dimensionless and is actually another form of the dimensionless damping ratio $\zeta$. Once $\delta$ is known, $\zeta$ can be found by solving $\zeta=\delta / \sqrt{(2 \pi)^{2}+\delta^{2}}$, or it can be approximated as

$$
\zeta \cong \frac{\delta}{2 \pi}, \quad \text { if } \delta \ll 2 \pi \text { or } \zeta \ll 1 .
$$

If the damping in the given system is not known, it can be determined experimentally by measuring any two consecutive displacements $\mathrm{x}_{1}$ and $\mathrm{x}_{2}$ one cycle apart. By taking the natural logarithm of the ratio of $\mathrm{x}_{1}$ and $\mathrm{x}_{2}, \delta$ is obtained. By using (9), the damping ratio $\zeta$ can be computed. In fact, the damping ratio $\zeta$ can also be found by measuring two displacements separated by any number of complete cycles.

If $\mathrm{x}_{1}$ and $\mathrm{x}_{m+1}$ denote the amplitudes corresponding to times $\mathrm{t}_{1}$ and $t_{m+1}=t_{1}+m \tau_{d}$, where $\mathrm{m}$ is an integer, $x_{1} / x_{m+1}=$ $x_{1} / x_{2} \cdot x_{2} / x_{3} \cdot x_{3} / x_{4} \cdots x_{m} / x_{m+1}$. Any two consecutive displacements separated by one cycle satisfy the equation $x_{j} / x_{j+1}=e^{\zeta \omega_{n} \tau_{d}}$. So $x_{1} / x_{m+1}=\left(e^{\zeta \omega_{n} \tau_{d}}\right)^{m}=e^{m \zeta \omega_{n} \tau_{d}}$ and $\delta=1 / m \ln \left(x_{1} / x_{m+1}\right)$, which can be substituted into the damping ratio equation to obtain the viscous damping ratio $\zeta$. 


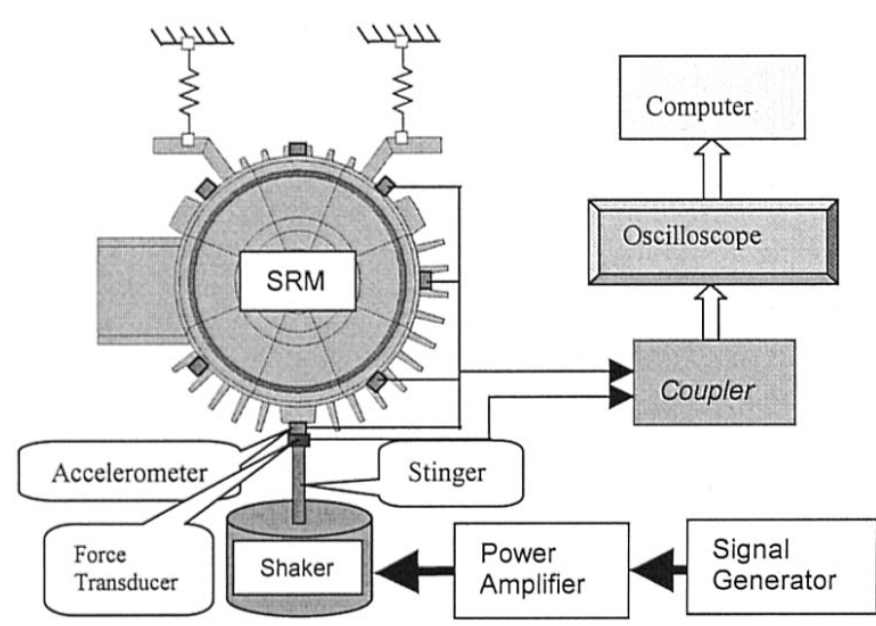

Fig. 3. Test setup of sinusoidal excitation method.

\section{IDENTIFICATION OF THE TRASFER FUNCTION USING THE SHAKER AND HAMMER}

\section{A. Test Setup and Procedure}

A shaker is used to measure the parameters of the transfer function for each resonant frequency. The test setup is shown in Fig. 3. An 8/6 4-kW SRM is suspended with four elastic ropes through its mounting holes to a solid bench, to establish a so-called free-free condition. A signal generator (Tektronix TM503, $3 \mathrm{MHz}$ ) supplies sinusoidal signals of different frequencies to the power amplifier (B\&K 2706). A shaker (B\&K 4809) is driven by the sinusoidal signals and excites the SRM. A force transducer is stud-mounted onto the body frame (through a threaded hole) and connected to the shaker by the stinger. The shaker is placed directly on the ground where the reaction force will be absorbed. Accelerometers are screwed onto the body frame at several different locations to pick up the acceleration. Force and acceleration outputs are recorded in a storage oscilloscope (LeCroy 9304 AM, QUAD $200 \mathrm{MHz}, 100 \mathrm{MS} / \mathrm{s}$ ) through a coupler (Kistler 5134), and then used in a computer program for data processing.

The frequency of the sinusoidal signal output from the signal generator is adjustable. It is changed till the predominant motion of the frame body is due to the desired mode of vibration. Firstly, the so-called wide-band sweeps are performed. Watching the response signal on an oscilloscope (or listening to the noise), a rise in the amplitude of response is detected where a narrow-band sweep is performed, using smaller frequency changes, in order to identify the frequency of the mode more accurately. The shaker is shut down and the decaying waveforms of force and acceleration are recorded.

\section{B. Damping Ratio Measurement}

When a so-called "pure" mode is excited, the damping ratio of the stator at the modal frequency can be measured from the envelope of the damped sinusoidal response. The shaker is shut off to simulate a damped response of the stator at the frequency of the tested resonant mode. Ideally, the stator should exhibit a damped sinusoidal response at all points, with a single frequency of vibration being the frequency of the excited mode. Fig. 4 is the recorded damped sinusoidal force and acceleration
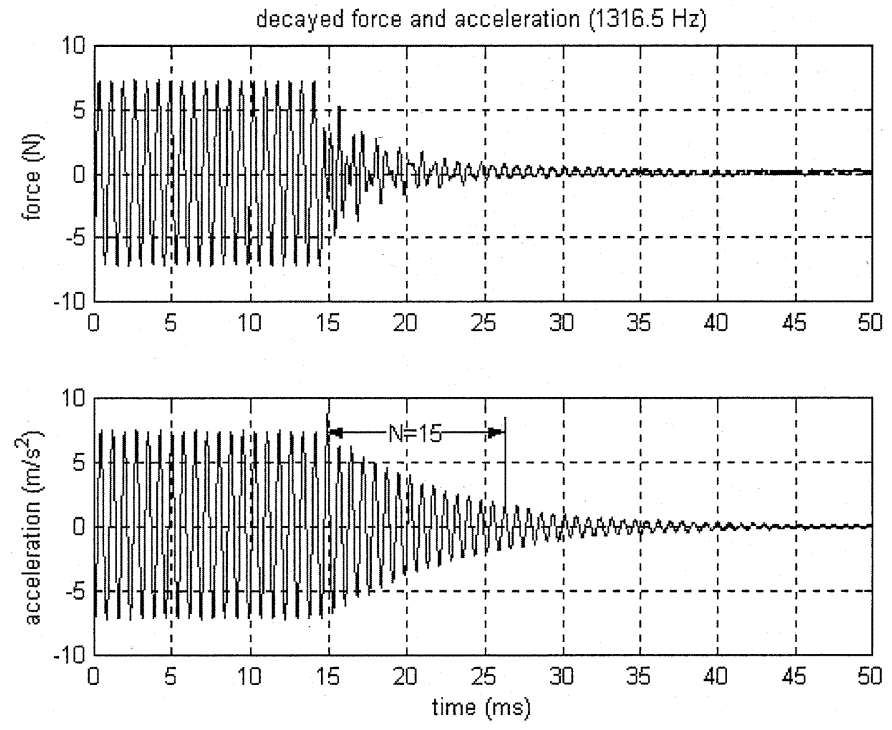

Fig. 4. Damped response at the resonant frequency.

responses after the shaker was shut off during excitation of the second mode.

From Fig. 4, the damping ratio of the second mode is calculated. Logarithmic decrement

$$
\delta=\frac{1}{N} \ln \frac{x_{k}}{x_{k+N}}=\frac{1}{15} \ln \frac{7.19235}{1.7602}=0.09384 .
$$

The damping ratio

$$
\zeta_{2}=\frac{\delta}{\sqrt{(2 \pi)^{2}+\delta^{2}}} \approx \frac{\delta}{2 \pi}=\frac{0.09384}{2 \pi} \approx 0.0156 .
$$

\section{Gain Relative to the Second Mode}

From

$$
\begin{aligned}
& \text { From } H_{2}(s)=\frac{a_{2}(s)}{F_{2}(s)}=A_{2} \frac{s^{2}}{s^{2}+2 \zeta_{2} \omega_{2 n} s+\omega_{n 2}^{2}} \\
& \left|H_{2}(j \omega)\right|=A_{2}\left|\frac{(j \omega)^{2}}{(j \omega)^{2}+2 \zeta_{2} \omega_{n 2}(j \omega)+\omega_{n 2}^{2}}\right| \\
& =A_{2} \frac{\omega^{2}}{\sqrt{\left(\omega_{n 2}^{2}-\omega^{2}\right)^{2}+\left(2 \zeta_{2} \omega_{n 2} \omega\right)^{2}}} .
\end{aligned}
$$

At the resonant frequency,

$$
\left|H_{2}\left(j \omega_{n 2}\right)\right|=A_{2} \frac{\omega_{n 2}{ }^{2}}{\sqrt{\left(\omega_{n 2}^{2}-\omega_{n 2}^{2}\right)^{2}+\left(2 \zeta_{2} \omega_{n 2} \omega_{n 2}\right)^{2}}}=\frac{A_{2}}{2 \zeta_{2}} \text {. }
$$

From Fig. 4, the amplitude of the force is $7.1747 \mathrm{~N}$ (the sensitivity of the force transducer is $0.940 \mathrm{mV} / \mathrm{lbf}(1 \mathrm{lbf}=$ $4.4498 \mathrm{~N}$ ), and the coupler gain is 100), and the amplitude of acceleration is $7.2607 \mathrm{~m} / \mathrm{s}^{2}$ (the sensitivity of the accelerometers is $10.00 \mathrm{mV} / \mathrm{g}$, and the coupler gain is 100). Therefore,

$$
\left|H_{2}\left(j \omega_{n 2}\right)\right|=\left|\frac{a_{2}\left(j \omega_{n 2}\right)}{F_{2}\left(j \omega_{n 2}\right)}\right|=\frac{7.2607 \frac{\mathrm{m}}{\mathrm{s}^{2}}}{7.1747 \mathrm{~N}}=1.012 \mathrm{~kg}^{-1}
$$

The gain relative to the second mode is

$$
\begin{aligned}
A_{2} & =2 \zeta_{2}\left|H_{2}\left(j \omega_{n 2}\right)\right|=2 \times 0.0156 \times 1.012 \mathrm{~kg}^{-1} \\
& =0.0315744 \mathrm{~kg}^{-1} .
\end{aligned}
$$


TABLE I

PARAMETERS FOR THE SECOND- AND THIRD-ORDER VIBRATION TRANSFER FUNCTIONS

\begin{tabular}{c|c|c|c}
\hline Mode No. & Frequency $(\mathrm{Hz})$ & Damp Ratio $(\zeta)$ & Gain $\mathrm{A}\left(\mathrm{kg}^{-1}\right)$ \\
\hline 2 & 1316.5 & 0.0156 & 0.0315744 \\
\hline 3 & 2480.2 & 0.0241 & 0.0054461 \\
\hline
\end{tabular}

\section{Final Transfer Function}

Finally, the transfer function for the second mode of the SRM stator can be written as

$$
H_{2}(s)=A_{2} \frac{s^{2}}{s^{2}+2 \zeta_{2} \omega_{n 2} s+\omega_{n 2}^{2}}
$$

where $A_{2}=0.0315744 \mathrm{~kg}^{-1}, \zeta_{2}=0.0156, \omega_{n 2}=2 \pi f_{2}=$ $2 \pi \times 1316.5 \mathrm{rad} / \mathrm{s}$, and $s$ is the Laplace's variable.

Similarly, the transfer function of the other resonant frequencies can be obtained with the same method. The results of the second and third mode resonant frequencies are listed in Table I.

\section{WIDE-BAND EXCITATION EXPERIMENTS-IMPULSE HAMMER EXCITATION METHOD}

\section{A. Introduction}

Mechanical vibration modal tests for electric motors require a precise dynamic force measurement as well as corresponding acceleration measurement, although there is no theoretical restriction as to the waveform. Precise force measurement can be achieved by electrodynamic or servohydraulic exciters (vibrators) controlled by a signal generator (sinusoidal wave or even white noise signal) via a power amplifier, and a force transducer (load cell) between the exciter and the object to be tested. However, a more convenient and economical excitation method can be used-a hammer mounted with a high-quality piezoelectric force transducer at its tip. In applications like modal tests for middle-size electric motors, where a high crest factor and a limited ability to shape the input force spectrum is of no concern, modal hammer testing is an ideal excitation method. It is also an advantage for impact hammers that they are highly portable for fieldwork and provide no unwanted mass loading to the structure under test.

The modal hammer produces an impulse force at one hit, which excites the structure with a constant force over a frequency range of interest. It is also called nonattached broadband excitation. The waveform produced by a modal hammer is an impact-a transient (short duration) energy transfer event. The spectrum is continuous, with maximum amplitude at $0 \mathrm{~Hz}$ and decaying amplitude with increasing frequency. There are three interchangeable tips provided for the hammer, which determine the width of the input pulse and, thus, the bandwidth. Typical force impulse and its spectra produced with different tips are shown in Fig. 5. Different models of hammers together with their tips are compared carefully, the final goal is to excite the structure over the entire frequency range of interest simultaneously.

For the commercial hammer, its structure is acceleration compensated to avoid glitches in the spectrum due to hammer structure resonance, and the ergonomically designed handle

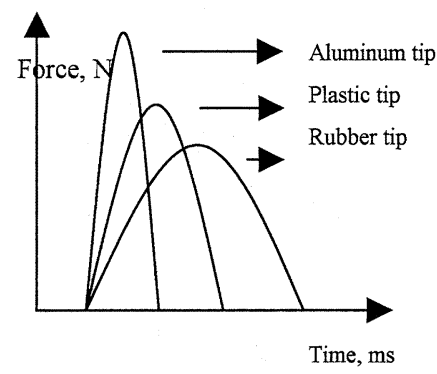

(a)

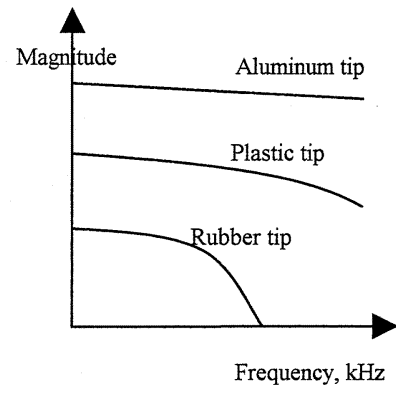

(b)
Fig. 5. Modal hammer impact force waveform and spectra. (a) Force waveform. (b) Force spectra.

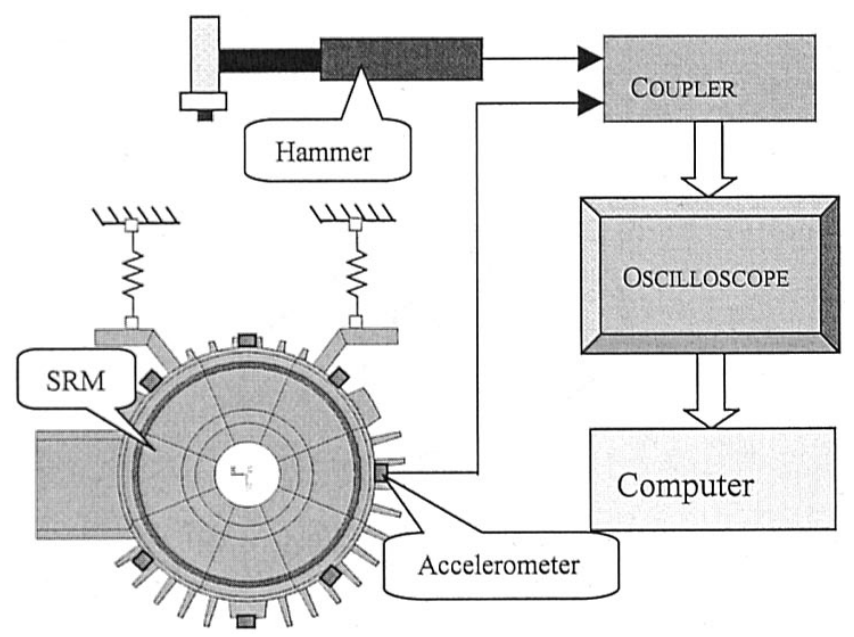

Fig. 6. Test setup of modal hammer excitation experiment.

grip helps the users optimize control and reduce the possibility of "double hits," which has to be avoided during the test.

\section{B. Hammer Excitation Setup}

The experimental system is shown in Fig. 6. A modal hammer (ENDEVCO 2302-10, 500-lb range, frequency range $8 \mathrm{kHz}$, sensitivity $10 \mathrm{mV} / \mathrm{lb}$ ) is used to supply an impulse force signal (broad-band force excitation). A coupler (or signal conditioner) (ENDEVCO 4416 B, or Kistler 5134) is used for force and acceleration signal conditioning.

\section{Hammer Excitation Procedure}

As can be seen in Fig. 6, the procedure for hammer excitation is much simpler than the shaker excitation method. The hammer can be moved anywhere for the tester's interests and convenience, which is sometimes impossible for the shaker. With one hit, information for all the frequencies of interest will be recorded and ready for analysis.

\section{Experimental Results}

1) Impulse Force and Acceleration Response in Time Domain: Fig. 7 shows the recorded impulse force and acceleration response in the time domain. As can be seen in the amplified view of the impulse force, it should be as sharp as possible. The sharpness (how close it is to a "perfect" impulse) depends on the hammer model, the tip material, the tested object, and also the experience of the tester. 

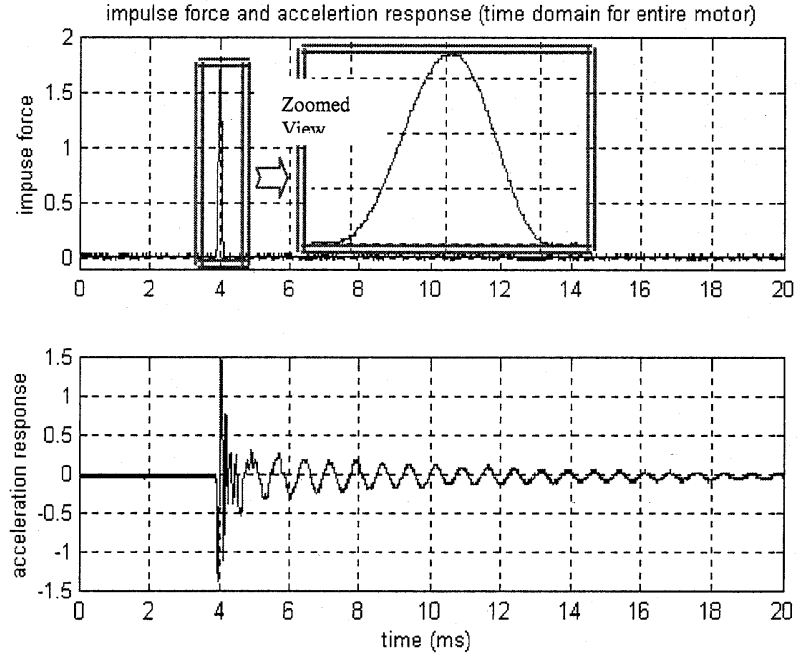

Fig. 7. Measured time-domain waveforms of force and acceleration.
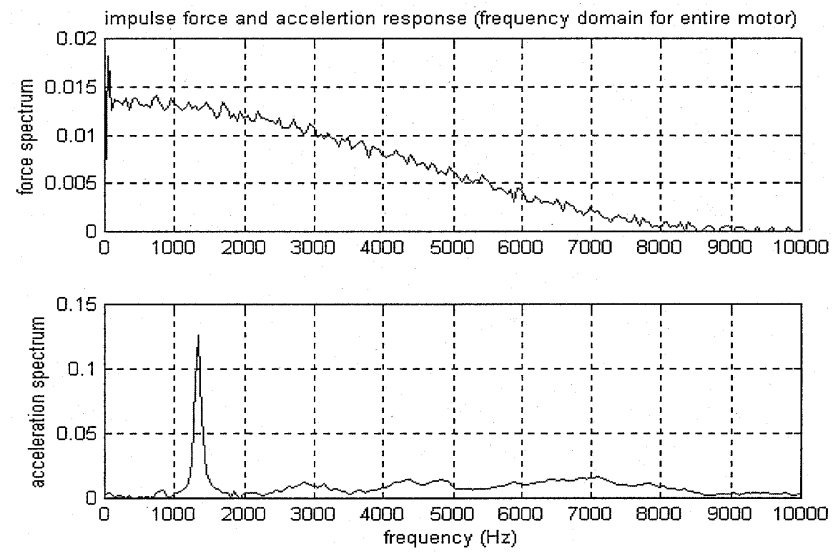

Fig. 8. Frequency-domain analysis of force and acceleration.

The acceleration waveform shows a decayed curve of acceleration (vibration). It is clear that there are several frequencies present because it shows "beating" phenomena.

2) Impulse Force and Acceleration Response in the Frequency Domain: Fig. 8 is the frequency-domain representation of the impulse force and acceleration responses. The impulse force has the information from $0 \mathrm{~Hz}$ up to $8 \mathrm{kHz}$, but the usable frequency range is $0 \mathrm{~Hz}-5 \mathrm{kHz}$. For the frequency range $5-8 \mathrm{kHz}$, it is not reliable, because the energy in this range is low, making it hard to excite any resonant mode frequencies.

It can also be seen from Fig. 8 that the peak response of acceleration is $1345 \mathrm{~Hz}$, which is the second mode resonant frequency for the entire motor (motor with end-bells and rotor). This result is very close to that of sinusoidal excitation and FEM results. The second mode vibration dominates the acceleration spectrum.

3) Transfer Function Calculation: Fig. 9 is the transfer function of the SRM from hammer excitation. A dominant peak is at $1345 \mathrm{~Hz}$, which is the second mode resonant frequency. The other resonant frequencies are not shown clearly. This is because they are low compared with the second mode, and the energy used in the hammer excitation test is low for the other excitations.

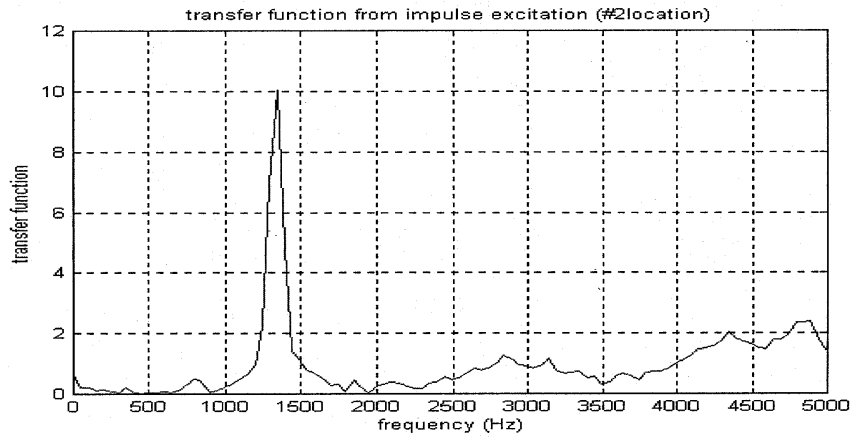

Fig. 9. Transfer function calculation of force to accleration.

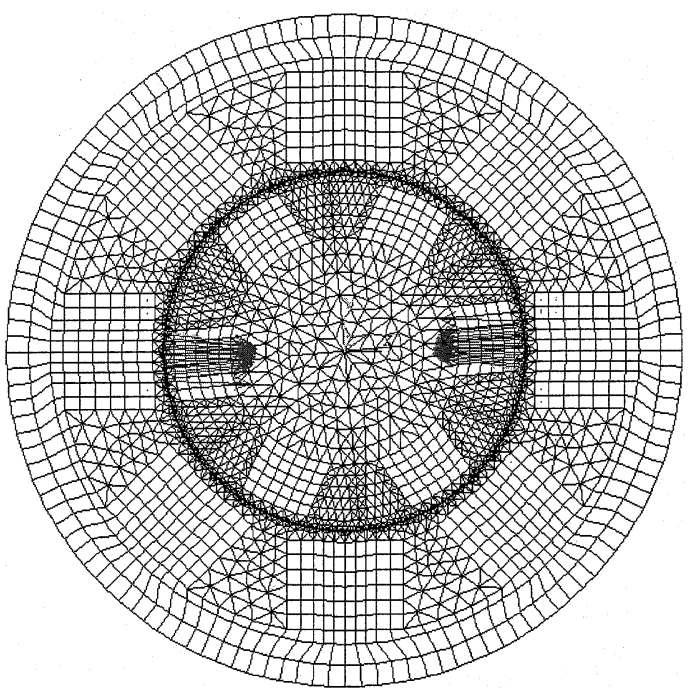

Fig. 10. FE meshing and force plot.

\section{CAlCulation OF THE NORMAl ForCE}

\section{A. Lookup Table Construction}

The normal force between SRM stator poles and rotor poles causes an attraction and thus ovalization of the stator, which is the main cause of SRM vibration and acoustic noise. The normal force in the SRM is very difficult to measure from experiment. Hence an FE package (ANSYS) is used here to calculate the normal force in SRMs.

A two-dimensional (2-D) ANSYS model is used for electromagnetic FE analysis [13], [14], as shown in Fig. 10. Static force calculations are carried out for 31 positions from aligned to unaligned (from $0^{\circ}$ to $30^{\circ}$, in steps of $1^{\circ}$ ), and at each position, 25 different currents (from 1 to $25 \mathrm{~A}$, in steps of $1 \mathrm{~A}$ ) are applied to the windings. At each position and current, the normal force and tangential force (which leads to torque) are calculated and a table of force versus rotor position and phase current is constructed. Fig. 11 shows the force plot of the SRM as a function of phase current and rotor position.

This normal force versus phase current and rotor position lookup table can now be used to obtain the actual normal force acting on the stator, for an arbitrary phase current waveform, once turn-on and turn-off angles are known. This will be used to determine the force harmonics and, hence, to calculate the vibrations resulting from the normal forces. 


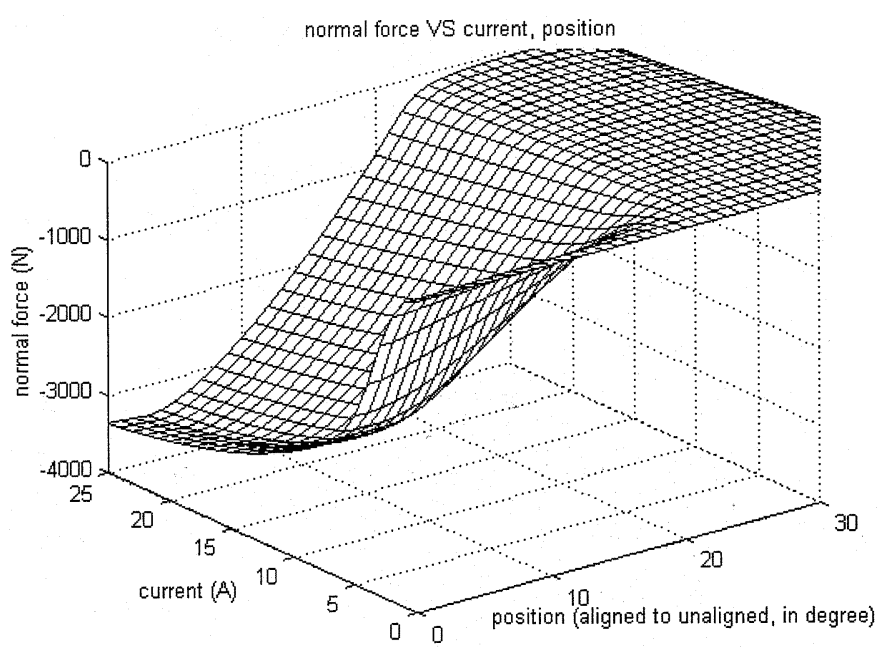

Fig. 11. Plot of normal force versus phase current and rotor position.

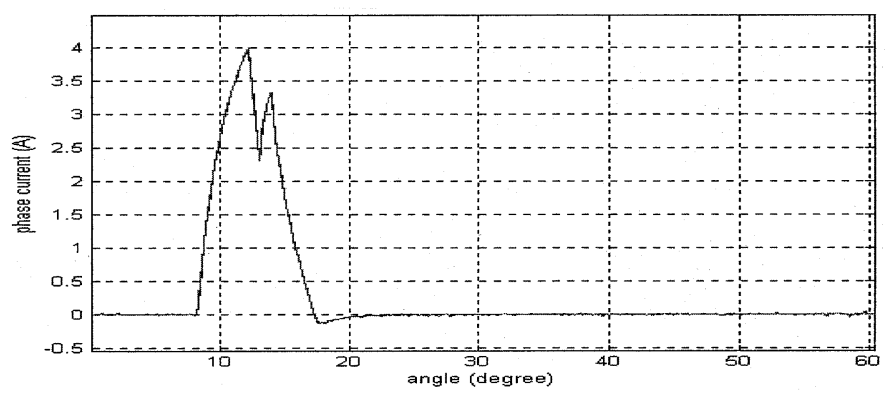

Fig. 12. Measured SRM phase current at $1450 \mathrm{r} / \mathrm{min}$.

\section{PREDiction of VibRation IN THE SRM}

\section{A. Vibration Prediction}

Fig. 12 shows the recorded phase current waveform of the SRM at $1450 \mathrm{r} / \mathrm{min}$ (no load). With the known turn-on and turn-off angles, this current waveform can be used in the previous lookup table to calculate the normal force acting on the stator poles, as shown in Fig. 13.

A fast Fourier transform (FFT) calculation is carried out for the above normal force as shown in Fig. 14. The frequency of the fundamental is

$$
f_{1}=\frac{1450 \mathrm{r} / \mathrm{min}}{60 \frac{\mathrm{s}}{\mathrm{min}}} \times 6 \text { poles }=145 \frac{\text { revolution }}{\mathrm{s}}=145 \mathrm{~Hz} \text {. }
$$

The normal force magnitudes of all the harmonics are shown in this figure together with the frequencies. This result can be used as input to the simulation model described in the previous paragraph; thus, the output will be the simulated acceleration response. Fig. 15 shows the input (normal force) and output (stator acceleration) in the vibration prediction model, in both time and frequency domain.

\section{Correlation With Test Results}

\section{A. Running Motor Test Results}

Fig. 16 shows the test setup for the SRM acceleration experiment. The SRM is controlled by a TASC Drive (OULTON Switched Reluctance Drive), with which the speed is adjustable. The SRM is suspended by four ropes. Acceleration responses

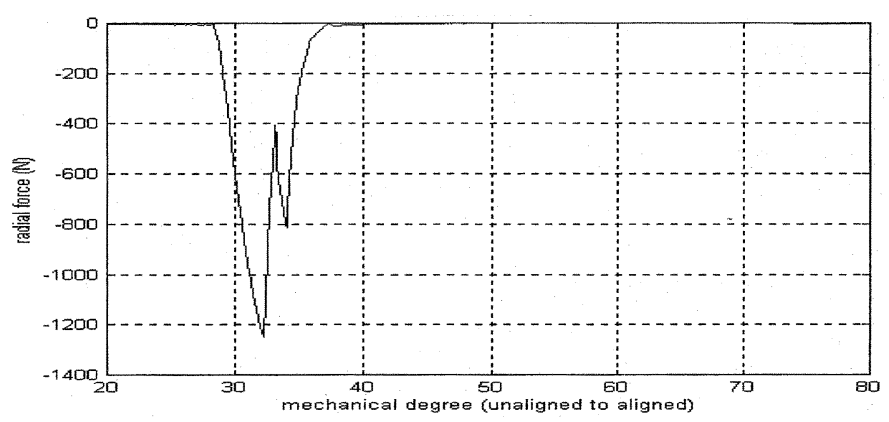

Fig. 13. Normal force calculated from lookup table.

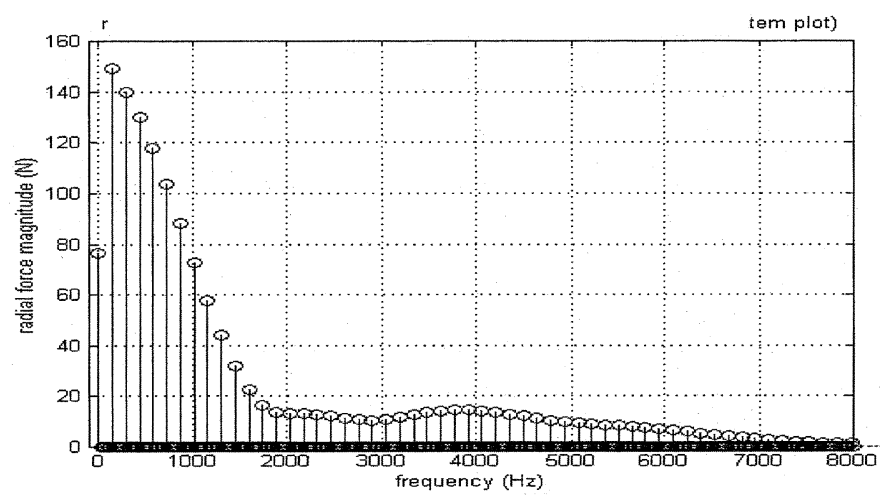

Fig. 14. FFT analysis results of normal force.
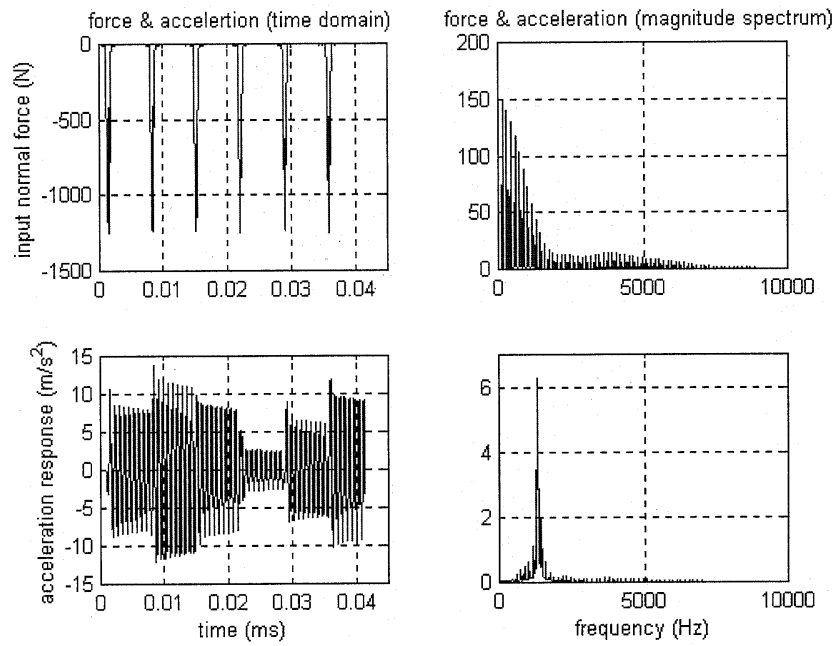

Fig. 15. Input normal force and output acceleration in vibration prediction model (simulated results at $1450 \mathrm{r} / \mathrm{min}$ ).

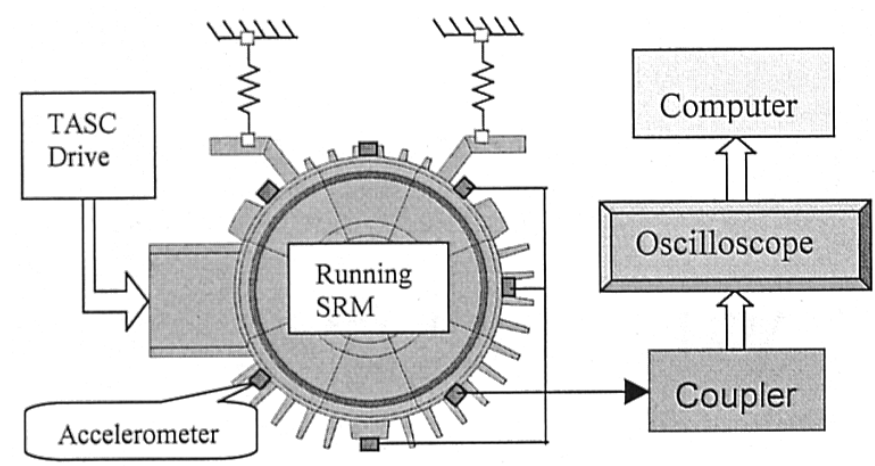

Fig. 16. Test setup of running SRM acceleration experiment. 

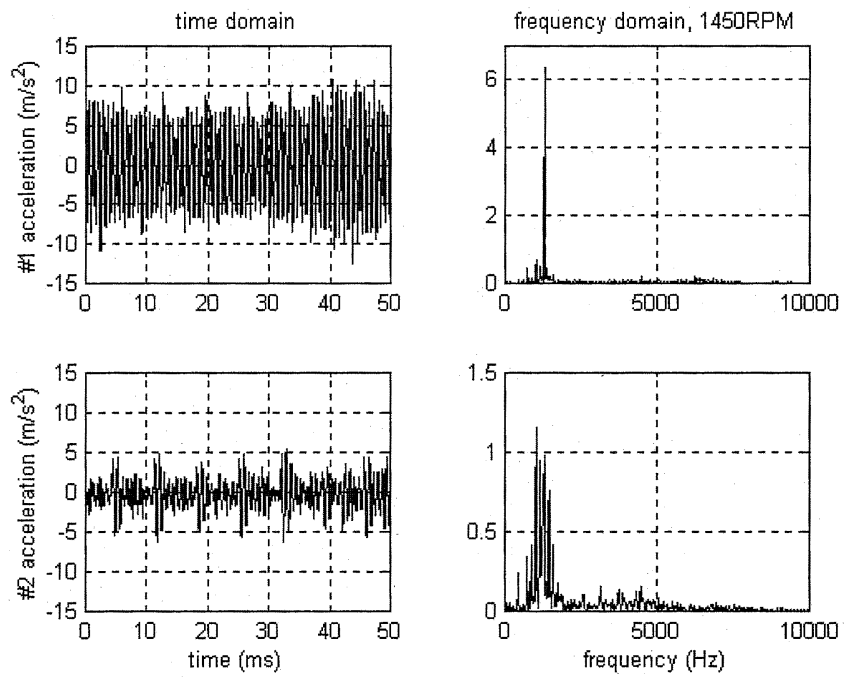

Fig. 17. Acceleration response in time and frequency domain (measures results at $1450 \mathrm{r} / \mathrm{min}$ ).

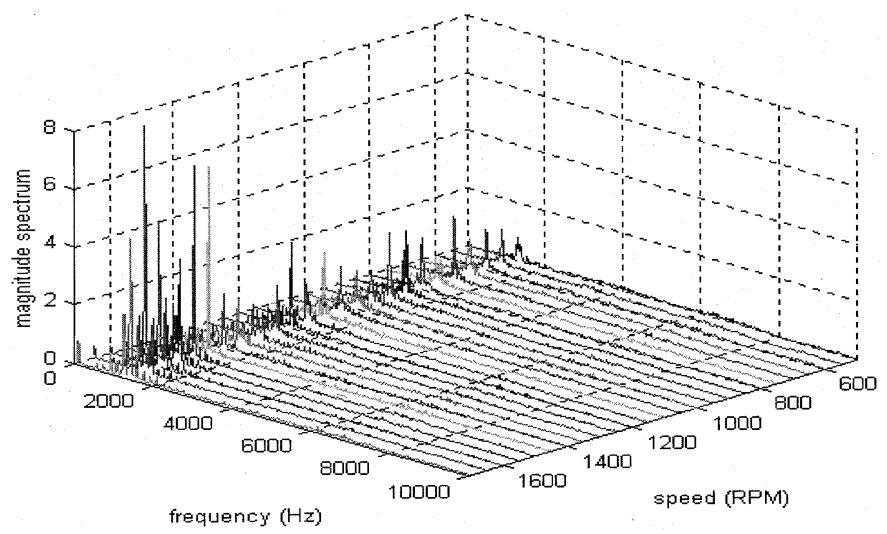

Fig. 18. 3-D view of acceleration spectra under different speeds.

from two different locations of the machine stator are recorded via the storage oscilloscope, from 500 to $1700 \mathrm{r} / \mathrm{min}$ in steps of $50 \mathrm{r} / \mathrm{min}$. Another storage oscilloscope is used to record the phase current waveform of the SRM; the current waveform can be used in the previous lookup table for the normal force.

Fig. 17 shows the time- and frequency-domain acceleration response at one of the selected 25 different speeds (1450 r/min). The frequency-domain response is more useful and straightforward. As seen in the figure, a peak can be easily observed. The responses under all the other speeds are recorded and analyzed in the same way, and are plotted in Fig. 18. This experiment is done under no load.

\section{B. Correlation With Predicted Results}

The measured stator acceleration, which is shown in Fig. 17, is very close to the predicted one shown in Fig. 15. The frequency-domain response is close enough, which provides confidence that the vibration prediction model based on vibration test and FE calculations is valid.
Fig. 17 also shows the measured acceleration at a different location from peak of the second-order mode shape, which is very low. Fig. 18 shows that the second-order resonant frequency dominates all the others at all speeds for this motor.

\section{CONCLUSIONS}

A normal force-stator acceleration transfer function is determined in this paper based on shaker excitation and impulse hammer tests. A detailed normal force versus phase current and rotor position lookup table is constructed using FE calculations. A vibration prediction model of the SRM is built based on the transfer function and normal force lookup table. The model is then verified by a running motor test, which shows acceptable accuracy. This now allows the possibility of using a transfer function model such as this for improved design of SRMs from a vibration and acoustic noise point of view.

\section{REFERENCES}

[1] D. E. Cameron, J. H. Lang, and S. D. Umans, "The origin and reduction of acoustic noise in doubly salient variable-reluctance motors," IEEE Trans. Ind. Applicat., vol. 28, pp. 1250-1255, Nov./Dec. 1992.

[2] R. S. Colby, F. Mottier, and T. J. E. Miller, "Vibration modes and acoustic noise in a 4-phase switched reluctance motor," in Conf. Rec. IEEE-IAS Annu. Meeting, vol. 1, Orlando, FL, Oct. 8-12, 1995, pp. 441-447.

[3] W. Cai, P. Pillay, and A. Omekanda, "An analytical model to predict the modal frequencies of switched reluctance motors," in Proc. IEEE IEMDC, Boston, MA, June 2001, pp. 203-207.

[4] P. Pillay and W. Cai, "Investigation into vibration in switched reluctance motor," IEEE Trans. Ind. Applicat., vol. 35, pp. 589-596, May/June 1999.

[5] W. Cai and P. Pillay, "Resonant frequencies and mode shapes of switched reluctance motors," IEEE Trans. Energy Conversion, vol. 16, pp. 43-48, Mar. 2001.

[6] C. Y. Wu and C. Pollock, "Time domain analysis of vibration and acoustic noise in the switched reluctance drive," in Proc. IEE Sixth Int. Conf. Electrical Machines and Drives, London, U.K., 1993, pp. $558-563$.

[7] - "Analysis and reduction of acoustic noise and vibration in the switched reluctance drive," IEEE Trans. Ind. Applicat., vol. 31, pp. 91-98, Jan./Feb. 1995.

[8] W. Cai, P. Pillay, Z. Tang, and A. Omekanda, "Vibration measurements in the switched reluctance motor," presented at the IEEE-IAS Annu. Meeting, Chicago, IL, Sept. 30 -Oct. 4, 2001.

[9] S. P. Verma and R. S. Girgis, "Method for accurate determination resonant frequencies and vibration behavior of stators of electrical machines," Proc. Inst. Elect. Eng., pt. B, vol. 128, no. 1, pp. 1-11, Jan. 1981.

[10] R. S. Girgis and S. P. Verma, "Experimental verification of resonant frequencies and vibration behavior of stators of electrical machines, part I-Models, experimental procedure an apparatus," Proc. Inst. Elect. Eng., pt. B, vol. 128, no. 1, pp. 12-21, Jan. 1981.

[11] S. R. Singiresu, Mechanical Vibrations. Reading, MA: Addision-Wesley, 1995.

[12] R. C. Stroud, "Excitation, measurement, and analysis methods for modal testing," Sound Vibration, pp. 12-27, Aug. 1987.

[13] T. J. E. Miller, Switched Reluctance Motors and Their Control. Oxford, U.K.: Magna Physics/Clarendon, 1993.

[14] R. Arumugam, D. A. Lowther, R. Krishnan, and J. F. Lindsay, "Magnetic field analysis of a switched reluctance motor using a two dimensional finite element method," IEEE Trans. Magn., vol. 21, pp. 1883-1885, Sept. 1985.

[15] J. Mahn, D. Williams, P. Wung, G. Horst, J. Llooyd, and S. Randall, "A systematic approach toward studying noise and vibration in switched reluctance machines: Preliminary results," in Conf. Rec. IEEE-IAS Annu. Meeting, San Diego, CA, Oct. 6-10, 1996, pp. 779-785. 


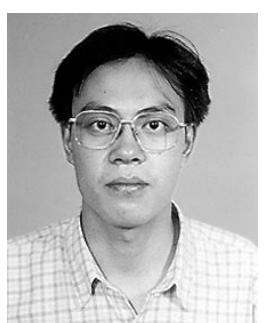

Zhangjun Tang (S'00-M'03) received the B.S. degree in electrical engineering from Harbin Institute of Technology, Harbin, China, in 1994, the M.S. degree from Beijing Institute of Control Devices, Beijing, China, in 1997, and the Ph.D. degree from Clarkson University, Potsdam, NY, 2002.

Following receipt of the M.S. degree, he spent $21 / 2$ additional years at Beijing Institute of Control Devices, developing gyroscope motor and control systems for various navigation platforms. In September 2002, he joined Stryker Instruments, Kalamazoo, MI, as a Senior Design Engineer in the Powertools Product Platform Research and Development Group. His research interests include design, control, and modeling of electrical machines and drives, numerical computation of electromagnetic fields and mechanical structures, and vibration and noise of electrical machines.

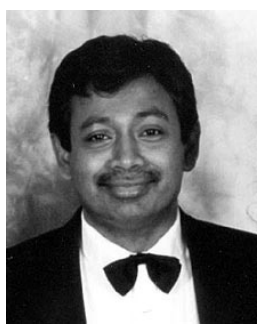

Pragasen Pillay (S'84-M'87-SM'92) received the Bachelor's degree from the University of Durban-Westville, Durban, South Africa, in 1981, the Master's degree from the University of Natal, Durban, South Africa, in 1983, and the Ph.D degree from Virginia Polytechnic Institute and State University, Blacksburg, in 1987.

From January 1988 to August 1990, he was with the University of Newcastle-upon-Tyne, U.K. From August 1990 to August 1995, he was with the University of New Orleans. Currently, he is with Clarkson University, Potsdam, NY, where he is a Professor in the Department of Electrical and Computer Engineering and holds the Jean Newell Distinguished Professorship in Engineering. His research and teaching interests are in modeling, design, and control of electric motors and drives for industrial and alternate energy applications.

Dr. Pillay is a member of the IEEE Power Engineering, IEEE Industry Applications (IAS), IEEE Industrial Electronics, and IEEE Power Electronics Societies. He is a member of the Electric Machines Committee and Chairman of the Industrial Drives Committee of the IAS and Chairman of the Induction Machinery Subcommittee of the IEEE Power Engineering Society. He has organized and taught short courses in electric drives at IAS Annual Meetings. He is a member of the Institution of Electrical Engineers, U.K., and a Chartered Electrical Engineer in the U.K. He was also a recipient of a Fulbright Scholarship.

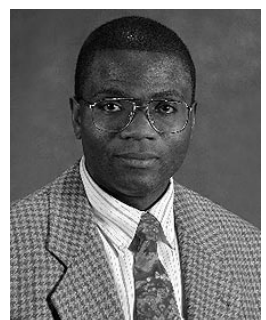

Avoki M. Omekanda (M'95-SM'97) received the Bachelor's degree in physics from Mohammed V University, Rabat, Morocco, in 1984, and the Engineering and $\mathrm{Ph} . \mathrm{D}$. degrees in electrical engineering from the Faculté Polytechnique de Mons, Mons, Belgium, in 1987 and 1993, respectively.

Following the receipt of the Engineer's diploma, he worked for the Research Laboratories of A.C.E.C Corporation, Charleroi, Belgium. In January 1990, he joined the Faculté Polytechnique de Mons as a Research Engineer. His research interests included computer-aided design of switched reluctance motors and magnetic field computation using munerical methods. After receiving the Ph.D. degree, he was an Assistant Professor in the Electrical Engineering Department, Faculté Polytechnique de Mons, for two years. In June 1995, he joined the General Motors Research and Development Center, Warren, MI, as a Senior Research Engineer. In 1999, he became part of the Delphi Research Labs, Shelby Township, MI, where he is currently a Staff Research Engineer. His research area includes design, analysis, and control of electric machines, in particular, switched reluctance, for automotive applications.

Dr. Omekanda is a Member of the Association des Ingénieurs de Mons (Belgium) and the Société des Electriciens et des Electroniciens (France). 